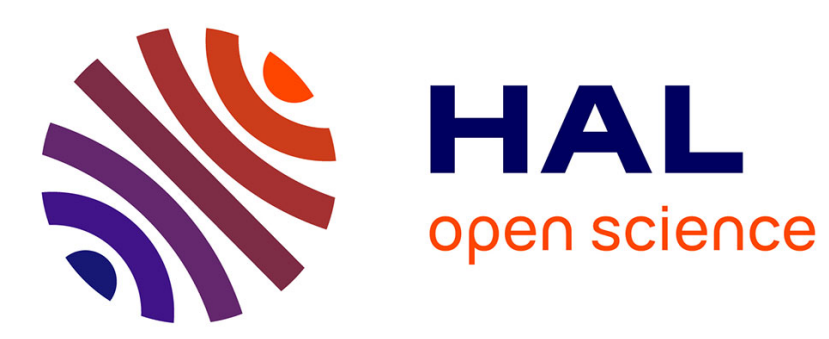

\title{
Malaisie, une relecture de la préhistoire et de l'histoire nationale avant Melaka \\ Daniel Perret
}

\section{To cite this version:}

Daniel Perret. Malaisie, une relecture de la préhistoire et de l'histoire nationale avant Melaka. Bulletin de l'Ecole française d'Extrême-Orient, 1995, 82 (1), pp.309 - 312. 10.3406/befeo.1995.2311 . halshs01880408

\section{HAL Id: halshs-01880408 \\ https://shs.hal.science/halshs-01880408}

Submitted on 24 Sep 2018

HAL is a multi-disciplinary open access archive for the deposit and dissemination of scientific research documents, whether they are published or not. The documents may come from teaching and research institutions in France or abroad, or from public or private research centers.
L'archive ouverte pluridisciplinaire HAL, est destinée au dépôt et à la diffusion de documents scientifiques de niveau recherche, publiés ou non, émanant des établissements d'enseignement et de recherche français ou étrangers, des laboratoires publics ou privés. 


\section{Malaisie, une relecture de la préhistoire et de l'histoire nationale} avant Melaka

Daniel Perret

\section{Citer ce document / Cite this document :}

Perret Daniel. Malaisie, une relecture de la préhistoire et de l'histoire nationale avant Melaka. In: Bulletin de l'Ecole française d'Extrême-Orient. Tome 82, 1995. pp. 309-312;

doi : https://doi.org/10.3406/befeo.1995.2311

https://www.persee.fr/doc/befeo_0336-1519_1995_num_82_1_2311

Fichier pdf généré le 07/05/2018 


\section{MALAISIE}

\section{Une relecture de la préhistoire et de l'histoire nationale avant Melaka}

En 1994 (le 14 avril et le 18 octobre), Arkib Negara Malaysia (les Archives Nationales de Malaisie) a été le cadre d'une série de deux forums intitulés «Polemik Sejarah Malaysia; Sejarah Malaysia sebelum 1400: kesinambungan dan perubahan ». Ouverte au public et aux journalistes (à qui l'on a toutefois interdit la publication de tout compte rendu), chacune de ces deux sessions a été suivie par environ 300 auditeurs, en majorité étudiants de différentes universités de Kuala Lumpur.

L'objectif officiel affiché pour ces deux événements tenait en six points: faire prendre conscience de l'importance d'une connaissance de l'histoire du pays qui soit précise et juste, faire connaître les derniers développements de la recherche historique et archéologique concernant la Malaisie, fournir informations et données historiques présentant un intérêt pour l'histoire nationale, inciter les chercheurs à utiliser les sources primaires, former une société détentrice de savoir, développer un esprit patriotique.

Chaque session a été animée par trois intervenants principaux : Prof. Dato' Dr. Nik Hassan Shuhaimi, archéologue enseignant à Universiti Kebangsaan Malaysia (présent aux deux sessions); Prof. Dato' Siti Zuraina, préhistorienne enseignant à Universiti Sains Malaysia (présente à la première session); Mr Adi bin Haji Taha, archéologue au musée national (présent aux deux sessions); Prof. Dato' Dr. Wan Hashim bin Wan Teh, directeur de Institut Alam dan Tamadun Melayu à Universiti Kebangsaan Malaysia (présent à la seconde session).

Comme l'indique le titre, ces forums ont été consacrés à l'histoire de la Malaisie avant l'apparition de Melaka et ont mis nettement en lumière les critiques, sinon le rejet, par un certain nombre de chercheurs locaux de théories et d'hypothèses formulées par les historiens et archéologues occidentaux. Ainsi, aux théories sur les migrations et l'indianisation des seconds qui mettraient l'accent sur des facteurs externes pour expliquer les transformations socio-culturelles anciennes dans la péninsule malaise, les premiers opposent l'idée d'un développement autochtone où les facteurs externes n'auraient joué qu'un rôle périphérique. Á l'occasion de ces deux forums, des éléments nouveaux ont été avancés par les chercheurs présents pour étayer cette nouvelle vision de la préhistoire et de l'histoire ancienne de la Malaisie. Nous rapportons ci-dessous les principales théories et hypothèses énoncées par les chercheurs malaysiens présents. 


\section{Contre la «colonisation» par des migrations préhistoriques}

Le concept de «culture hoabinhienne» appliqué par les Occidentaux à une industrie lithique qui, en péninsule malaise, daterait de moins de 10000 ans, est réfuté. L'utiliser serait reconnaître que la «culture malaise vient de Hoa-Binh », apportée par une vague migratoire. Or, selon le professeur Zuraina, d'une part on ne peut réduire la culture aux outils, d'autre part elle a trouvé dans la province de Perak un site daté d'environ 30000 ans qui présente une industrie lithique semblable au hoabinhien et pourtant bien antérieure.

Les archéologues et historiens malaysiens refusent la théorie migratoire lancée il y a une centaine d'années et confortée par Heine-Geldern dans les années 1940: le peuplement de la péninsule malaise serait le résultat de trois vagues migratoires à partir du sud de la Chine, à savoir negritos il y a environ 5000 ans, proto-malaise il y a environ 2000 ans et deutéro-malaise il y a environ 1000 ans.

Pour le professeur Nik Hassan Shuhaimi, Orang Asli et Malais ont la même origine. L'image, couramment répandue, de Malais venant de l'étranger et repoussant les Orang Asli dans le plus profond de la forêt, doit être éliminée des livres d'histoire. En fait, la différenciation culturelle aurait eu lieu progressivement selon un ou des processus qui restent à déterminer. Le professeur Wan Hashim bin Wan Teh et Adi bin Haji Taha argumentent à ce propos en affirmant que des Orang Asli prétendent venir du Pays Minangkabau à Sumatra. D'autre part, pour montrer l'ancienneté de la présence de ces populations sur le sol de la péninsule, le professeur Zuraina rappelle qu'à la fin des années 1980, elle a mis au jour à Gua Gunung Runtuh près de Lenggong (Perak), le squelette d'un Homo sapiens qui serait vieux de 11000 ans (désigné depuis par l'expression «l'homme de Perak») et dont les caractéristiques seraient identiques à celles des Orang Asli actuels de la péninsule malaise. À la question d'un auditeur «L'homme de Perak est-il un orang Melayu? », le professeur Zuraina répondra que dans l'état actuel des connaissances, on ne peut raisonner qu'en terme d'anthropologie physique.

Un dernier élément est récemment venu s'ajouter aux précédents pour réfuter l'ancienne théorie des vagues migratoires originaires du Yunnan. Un groupe d'une centaine de chercheurs malaysiens s'est rendu en août 1994 dans cette région. Parmi eux figurait le professeur Wan Hashim bin Wan Teh qui, lors de la seconde session, a confirmé que d'après les données ethnographiques et linguistiques recueillies à l'occasion de cette visite, il n'existe aucune preuve claire pouvant confirmer la théorie selon laquelle la «ras Melayu» est originaire du Yunnan. En effet, aucun élément examiné au Yunnan n'a montré une quelconque similitude avec des éléments «keMelayuan». Prenant l'exemple de migrations ayant eu lieu depuis environ un millénaire, le professeur Wan Hashim montre qu'il reste toujours un groupe témoin sur le lieu d'origine. Aucune minorité ethno-linguistique du Yunnan ne possédant de liens linguistique ou culturel avec la famille (rumpun) malayo-polynésienne, il est difficile, selon lui, de croire que cette région a été le berceau de la «famille melayu». De plus, ajoute-t-il, deux autres facteurs vont contre l'idée d'une migration à partir du Yunnan : d'une part le Yunnan est une région plutôt fermée géographiquement, d'autre part c'est une zone fertile. Il n'y a donc pas de facteurs naturels incitant à quitter ce territoire.

Une théorie plus récente (soutenue en particulier par le professeur Peter Bellwood, présent dans la salle lors de la première session), fondée notamment sur des recherches linguistiques, décrit une migration austronésienne en provenance de Taiwan vers la péninsule malaise entre 2000 et 500 avant J.-C. 
Théorie perçue comme «romantique et spéculative» (Prof. Nik Hassan Shuhaimi), elle n'emporte pas la conviction des chercheurs locaux pour qui les preuves avancées ne sont pas claires.

Le professeur Bellwood réagit en maintenant que des mouvements de population ont bien eu lieu vers la Malaisie, que les données de l'archéologie sont ambiguës et ne donneront pas de réponses définitives. Il rappelle aux archéologues qu'ils ne doivent pas oublier la linguistique et ne pourront de toute manière jamais situer l'origine de la langue malaise. Selon le professeur Bellwood, les archéologues ne parviendront pas à résoudre à eux seuls la question de l'origine des Malais.

Enfin le professeur Zuraina avance l'hypothèse d'une production autochtone ancienne de métaux. Elle s'appuie sur le résultat d'une fouille menée dans les années 1980 à Gua Harimau (Lenggong, province de Perak) indiquant un niveau contenant un objet en bronze qui serait vieux de 5000 ans. Cette idée qui, selon elle, reste à conforter, invaliderait l'opinion généralement répandue que les métaux sont venus du nord à une date plus récente.

Pour conclure, ce n'est pas l'idée de migrations vers la péninsule malaise qui est contestée, mais les théories décrivant une «colonisation» par des migrations massives, théories qui pour l'instant sont jugées par les chercheurs locaux comme sans fondements sérieux. En fait ceux-ci ont tendance à penser que le cœur du monde malais (Alam Melayu) (du Vietnam à Java et de Sumatra à Bornéo) représente le lieu d'origine ('tempat asal' ou 'tanah ranah') de la «race " malayo-polynésienne. Toutefois, ils conçoivent que des preuves nouvelles recueillies avec des méthodes nouvelles, notamment des recherches interdisciplinaires, sont nécessaires pour reconstruire la véritable préhistoire de la région.

\section{Contre la colonisation indienne}

Depuis les publications de Quaritch-Wales dans les années 1940, l'idée selon laquelle les sites «indianisés» de Lembah Bujang à Kedah ont été l'œuvre de vagues de colons indiens depuis les premiers siècles de notre ère, reste profondément ancrée. Les chercheurs locaux refusent cette interprétation.

En fait, pour le professeur Nik Hassan Shuhaimi, les structures et objets découverts sont l'œuvre de «bumiputra» ayant adopté une culture venant d'Inde. Pour étayer sa théorie, il avance les arguments suivants:

- Les grandes différences dans le domaine religieux entre la péninsule malaise et l'Inde. Ainsi la statuaire, l'art et l'architecture des structures religieuses seraient différents de ceux rencontrés en Inde.

- À l'intérieur du monde malais même, la statuaire présente des différences: Kedah est différent de Palembang, lui-même différent de Jambi.

Le professeur Nik Hassan Shuhaimi interprète ces différences comme le signe d'un développement local autochtone. Selon lui, si les Indiens étaient les auteurs de ces productions, la statuaire serait identique partout.

En guise d'exemple, il montre que dans la statuaire de Bouddha à Dvaravati les visages sont môns, alors qu'à Java les visages sont indonésiens.

- Les documents épigraphiques tendraient également à prouver un développement autochtone. Alors que les signes sont indiens, la langue généralement utilisée est locale: malais ancien, javanais ancien, môn ou khmer.

Le Professeur Nik Hassan Shuhaimi conclut en affirmant qu'il ne faut pas voir l'indianisation comme un processus passif, mais au contraire comme le résultat d'un choix des sociétés indigènes. 


\section{Controverse sur le niveau culturel des indigènes de la préhistoire}

Les préhistoriens locaux remettent en cause l'idée qui serait répandue en Occident d'un lien entre le degré de sophistication des objets travaillés et le niveau culturel de leurs auteurs. S'il est indéniable que les pierres taillées européennes sont plus sophistiquées que les choppers de Malaisie, la raison est à chercher avant tout dans un environnement différent. Dans les zones tropicales, des besoins différents entraîneraient la création d'industries lithiques adaptées au milieu, différentes des industries lithiques européennes. Ainsi, il y a environ 30000 ans, les habitants de Kota Tampan (province de Perak) faisaient des outils efficaces et adaptés à leur milieu.

\section{Une vision eurocentrique de la périodisation de la préhistoire}

En Malaisie, les changements climatiques ont été beaucoup moins importants qu'en Europe. Les modifications sensibles ont surtout concerné le niveau des mers. Cette stabilité des conditions climatiques rend inadéquate, en Asie du Sud-Est, l'utilisation du terme 'mésolithique' qui, en Europe, indique la période d'adaptation à de nouvelles conditions climatiques.

Pour conclure sur ces deux forums marqués par la revendication d'un monde malais comme racine de sa propre histoire, il convient de les replacer dans un contexte plus large. Cette volonté d'ancrage sur ce qui serait un vieux fonds culturel autochtone est certainement révélatrice d'une crise identitaire qui frappe les Malais à un moment où le pays connaît de profonds bouleversements économiques et sociaux. Cette affirmation d'une spécificité est peut-être aussi à mettre en rapport avec le rôle phare que souhaite jouer la Malaisie dans l'ASEAN au début du troisième millénaire.

Daniel PERRET

\section{CORÉE}

\section{Réflexions sur une exposition.}

Inaugurée le 1er décembre 1994 à Seoul, dans l'enceinte du palais Kyŏngbok, une exposition sur les vestiges de la civilisation du royaume de Koguryŏ (Ier siècle av. J.-C. - VIIe siècle apr. J.-C.) a connu un immense succès populaire et a dû être prolongée de deux semaines, jusqu'au 16 avril. Pour beaucoup de Coréens, cela semble une découverte, presqu'un choc, qui réveille en eux des sentiments mêlés et rarement exprimés. Ces restes, disséminés à travers la Corée du Nord et la Mandchourie orientale, n'étaient certes pas tout à fait inconnus, mais avant la seconde guerre mondiale, c'était sous la direction de savants japonais qu'avaient été opérés explorations et relevés, plus d'ailleurs en Corée du Nord qu'en Mandchourie. Il y avait eu ensuite la division du 